\title{
Insuficiencia renal oculta y prescripción de fármacos en pacientes hospitalizados
}

\author{
J. M. PEÑA PORTA, C. V. DE VERA FLORISTÁN', M. BUENO LOZANO² \\ Unidad de Nefrología. Servicio de Medicina Interna. Hospital de Barbastro. Huesca. \\ ${ }^{1}$ Hospital Universitario Arnau de Vilanova. Lleida. ${ }^{2}$ Centro de Salud Tudela Oeste. \\ Tudela. Navarra
}

\begin{abstract}
HIDDEN RENAL FAILURE AND DRUG PRESCRIPTION IN HOSPITALIZED PATIENTS
\end{abstract}

\section{RESUMEN}

Fundamento y objetivo: Con la utilización de fórmulas para el cálculo de la tasa de filtrado glomerular se puede detectar la presencia de la denominada Insuficiencia renal "oculta" (IRO) (coexistencia de cifras de creatinina sérica normales junto con un aclaramiento inferior a 60 $\mathrm{mL} / \mathrm{min} / 1,73 \mathrm{~m}^{2}$ ). El objetivo de este estudio fue analizar la prevalencia de IRO en pacientes hospitalizados y como ello repercute en la prescripción de fármacos.

Pacientes y método: Se incluyeron 567 pacientes ingresados en la Sección de Medicina Interna del Hospital de Barbastro (Huesca) en un periodo de tres meses (abril-junio de 2006). Se cálculo el FG aplicando la fórmula MDRD abreviada. Mediante regresión logística se analizaron los factores asociados a la presencia de IRO. Se registraron los fármacos potencialmente peligrosos prescritos en el informe de alta.

Resultados: La prevalencia de IRO se situó en el 10,8\%. El 35,2\% de la muestra presentaba un estadio de enfermedad renal crónica grado 3,4 ó 5 según los criterios de la National Kidney Foundation (NKF). Las variables que se asociaron con la presencia de IRO fueron la edad $(\operatorname{expB}$ $=1,035 ;$ IC95\% $1,012-1,060 ; \mathrm{p}=0,004)$ y el sexo femenino $(\operatorname{expB}=$ 4,669; IC95\% 2,461 - 8,856; $\mathrm{p}<0,0001)$. En sólo el 1,8\% de los pacientes se calculó el aclaramiento de creatinina durante el ingreso. Un número considerable de pacientes con IRO recibieron tratamiento con fármacos potencialmente perjudiciales dado su grado de función renal.

Conclusiones: Nuestros resultados apoyan que se introduzca el filtrado glomerular estimado mediante la fórmula MDRD en los informes de los laboratorios como medida de la función renal.

PALABRAS CLAVE: Insuficiencia renal oculta. Prescripción de fármacos.

\section{ABSTRACT}

Objetive: Equations used to estimate glomerular filtarion rate $(G F R)$ are useful to detect "hidden" renal failure $(R F)$ ( coexistence of normal serum creatinine together with GFR $\left.<60 \mathrm{ml} / \mathrm{mto} / 1.73 \mathrm{~m}^{2}\right)$. The aim of this study was to anayze the prevalence of this phenomenon in hospitalized patients and how this affects drugs prescription.

Patients and method: 567 patients admitted to the internal medicine section of Barbastro Hospital (Huesca, Spain) during a three months period (april-june 2006) were included. GFR was estimated applying abreviated MDRD equation. By means of logystic regresion analysis we analyzed the factors associated with the presence of "hidden" $R F$. We also recorded the potentialy dangerous drugs prescibed at discharge from hospital.

Results: Prevalence of "Hidden" RF was $10.8 \%$. NKF cronic kidney disease stages 3.4 or 5 were present in $35.2 \%$ of patients. Variables asociated with presence of "hidden" $R F$ were age $(\exp B=1.035$; IC95\% 1.012-1.060; $p=0.004)$ and female sex (expB $=4.669 ;$ IC95\% $2.461-8.856 ; p<0.0001)$. Only in $1.8 \%$ of cases GFR was calculated during hospitalitation period. A considerable number of patients with "hidden" $R F$ received treatment with potentialy dangerous drugs taking into account his degree of renal failure.

Conclusions: Our results support the introduction of GFR estimation by means of MDRD equation in laboratory reports.

KEY WORDS: Hidden renal failure. Drug prescription.

Peña Porta JM, De Vera Floristan CV, Bueno Lozano M. Insuficiencia renal oculta y prescripción de fármacos en pacientes hospitalizados. An Med Interna (Madrid) 2007; 24: 221-226.

\section{INTRODUCCIÓN}

La enfermedad renal crónica (ERC) es considerada hoy en día un problema de salud pública dada su progresiva incidencia y prevalencia (1). En las poblaciones de riesgo se recomienda para su detección la medición de la albuminuria como marcador de daño renal y la estimación del filtrado glomerular
(FG) (2). Para esto último es útil la utilización de alguna ecuación basada en el nivel de creatinina sérica sin precisar la recogida de una muestra de orina de 24 horas que en muchas ocasiones resulta engorrosa y fuente de errores. Además de la ya clásica fórmula de Cockcroft-Gault (3) en los últimos años se ha introducido la denominada fórmula MDRD que en su forma abreviada es la recomendada por las guías para la esti-

Trabajo aceptado: 22 de enero de 2007 
mación del FG (4,5). Con la aplicación de estas fórmulas se ha puesto de manifiesto la existencia de un importante segmento de pacientes afectos de la denominada "insuficiencia renal oculta" (IRO) (6), es decir, pacientes con niveles séricos de creatinina dentro del rango del normalidad pero que presentan ya unos niveles de FG inferiores a $60 \mathrm{ml} / \mathrm{mto} / 1,73 \mathrm{~m}^{2}$. Este fenómeno se produce sobretodo en personas de edad avanzada ya que suele existir una sobrestimación de la función renal cuando esta se evalúa a través de la creatinina sérica. Se han llevado a cabo diversos estudios para determinar la prevalencia de este problema , fundamentalmente en atención primaria (6-12), pero hasta la fecha son menos los efectuados a nivel hospitalario. Por otra parte, una de las consecuencias del desconocimiento de la existencia de una insuficiencia renal es la posible prescripción inadecuada a los pacientes de determinados fármacos cuya dosificación debe ajustarse a los niveles de función renal o que deben manejarse con precaución en pacientes con fallo renal crónico $(13,14)$.

El objetivo del presente estudio es evaluar cual es la prevalencia de IRO dentro de un servicio hospitalario, los factores que influyen en su presentación y como ello repercute en la idoineidad de los fármacos prescritos a estos pacientes.

\section{MATERIAL Y MÉTODO}

Se incluyeron en el estudio todos los pacientes dados de alta en la sección de medicina interna del hospital de Barbastro (Huesca) en los meses de abril, mayo y junio de 2006. La sección incluye también las especialidades de nefrología, neumología, digestivo, cardiología, oncología y neurología. No se excluyó a los pacientes que fueron éxitus si presentaron una función renal estable durante el ingreso. Por el contrario los pacientes que presentaron un fracaso renal agudo fueron excluidos. Los reingresos fueron considerados como casos nuevos. Se revisó el informe de alta de cada paciente a través de la Intranet del hospital. De este informe se obtuvieron los siguientes datos: edad, sexo, creatinina sérica (de no figurar en el informe se obtuvo a través de la Intranet accediendo a

\section{TABLA I}

CARACTERÍSTICAS DE LOS PACIENTES AGRUPADOS SEGÚN FUNCIÓN RENAL

\begin{tabular}{|c|c|c|c|c|}
\hline & $I R C$ & $\begin{array}{c}\text { IRO } \\
\text { más frecuentes }\end{array}$ & FR normal & Significación \\
\hline $\begin{array}{l}\text { Edad (años) } \\
\text { Sexo }\end{array}$ & $79,50 \pm 9,15$ & $78,11 \pm 9,32$ & $68,34 \pm 16,9^{*}$ & $p<0,0001$ \\
\hline $\begin{array}{l}\% \text { Varón/\% Mujer } \\
\text { Creatinina sérica }\end{array}$ & $49,3 / 50,7$ & $21 / 79$ & $58,9 / 41,1$ & $p<0,0001$ \\
\hline$(m g / d l)$ & $2,19 \pm 1,6^{\#}$ & $1,10 \pm 0,11$ & $0,82 \pm 0,21^{\prime \prime}$ & $\begin{array}{l}{ }^{*} p<0,0001 \\
{ }^{*} p=0,048\end{array}$ \\
\hline Filtrado glomerular" & $36,48 \pm 13,4^{\#}$ & $55,01 \pm 3,2^{*}$ & $95,26 \pm 33,9$ & $\begin{array}{l}* p<0,0001 \\
" p<0,0001\end{array}$ \\
\hline Indice de comorbildad & $7,77 \pm 2$ & $7,18 \pm 2,02$ & $5,56 \pm 2,77^{*}$ & $p<0,0001$ \\
\hline $\begin{array}{l}N^{0} \text { de principios } \\
\text { activos prescritos }\end{array}$ & $6,62 \pm 3,03$ & $6,08 \pm 2,93$ & $4,54 \pm 2,69^{*}$ & $p<0,0001$ \\
\hline
\end{tabular}

los datos de laboratorio), antecedentes personales y diagnósticos al alta. Con los datos anteriores se calculó el índice de comorbilidad de Charlson (15). Se estimó asimismo el FG mediante la aplicación de la fórmula MDRD abreviada (5), que expresa el resultado del cálculo en $\mathrm{mL} / \mathrm{min} / 1,73 \mathrm{~m}^{2}$ :

$\left[\left(\mathrm{FG}=186 \mathrm{x} \mathrm{Cr}^{-1,154} \mathrm{x} \mathrm{edad}^{-0,203} \times 0,742\right.\right.$ (si sexo mujer) $\mathrm{x}$ 1,212 (si raza negra)]

Se registró el número y tipos de principios activos prescritos al alta. Se comprobó también si figuraban en el informe de alta una serie de fármacos potencialmente nefrotóxicos o cuya dosificación debe ser ajustada en casos de insuficiencia renal, incluidos en una lista previamente elaborada $(13,14)$ (Tabla II).

La creatinina sérica se determinó en el laboratorio mediante una modificación de la reacción cinética de Jaffé (método CREA, sistema Dimensión ${ }^{\circledR}$. Dade Behring Inc. UK). El límite normal de creatinina en nuestro hospital se sitúa en 1,3 $\mathrm{mg} / \mathrm{dl}$ en los hombres y en $1,1 \mathrm{mg} / \mathrm{dl}$ en las mujeres. Se definió como IRO la presencia de una creatinina sérica normal con un FG estimado inferior a $60 \mathrm{~mL} / \mathrm{min} / 1,73 \mathrm{~m}^{2}$. Se consideró "función renal normal" a la coincidencia de creatinina

\section{TABLA I}

LISTADO DE FÁRMACOS SELECCIONADOS QUE DEBEN SER AJUSTADOS SEGÚN LA FUNCIÓN RENAL O ADMINISTRADOS CON PRECAUCIÓN EN CASO DE

INSUFICIENCIA RENAL ADMINISTRADOS A LOS PACIENTES. SE AGRUPAN SEGÚN LA PRESENCIA DE IRC, IRO O FUNCIÓN RENAL NORMAL

\begin{tabular}{|c|c|c|c|c|}
\hline & & IRC & IRO & FR normal \\
\hline \multirow[t]{2}{*}{ Antiinflamatorios no esteroideos } & No & 131 & 57 & 343 \\
\hline & Sí & 7 & 4 & 23 \\
\hline \multirow[t]{2}{*}{ Amilorida } & No & 136 & 59 & 358 \\
\hline & Sí & 2 & 2 & 8 \\
\hline \multirow[t]{2}{*}{ Alopurinol } & No & 119 & 58 & 360 \\
\hline & Sí & 19 & 3 & 6 \\
\hline \multirow[t]{2}{*}{ Ranitidina, famotidina, cimetidina } & No & 137 & 60 & 364 \\
\hline & Sí & 1 & 1 & 2 \\
\hline \multirow[t]{2}{*}{ Aminoglucósidos } & No & 136 & 61 & 366 \\
\hline & Sí & 2 & 0 & 0 \\
\hline \multirow[t]{2}{*}{ Atenolol,nadolol,sotalol } & No & 132 & 61 & 364 \\
\hline & Sí & 6 & 0 & 2 \\
\hline \multirow[t]{2}{*}{ Biguanidas } & No & 136 & 57 & 358 \\
\hline & Sí & 2 & 4 & 8 \\
\hline \multirow[t]{2}{*}{ Ciprofloxacino,norfloxacino } & No & 137 & 58 & 357 \\
\hline & Sí & 1 & 3 & 9 \\
\hline \multirow[t]{2}{*}{ Colchicina } & No & 136 & 61 & 364 \\
\hline & Sí & 2 & 0 & 2 \\
\hline \multirow[t]{2}{*}{ Digoxina } & No & 119 & 45 & 334 \\
\hline & Sí & 19 & 16 & 32 \\
\hline \multirow[t]{2}{*}{ Espironolactona } & No & 131 & 54 & 352 \\
\hline & Sí & 7 & 7 & 14 \\
\hline \multirow[t]{2}{*}{ IECA/ARAII } & No & 82 & 39 & 284 \\
\hline & Sí & 56 & 22 & 82 \\
\hline \multirow[t]{2}{*}{ Levofloxacino } & No & 134 & 59 & 340 \\
\hline & Sí & 4 & 2 & 26 \\
\hline \multirow[t]{2}{*}{ Risperidona } & No & 134 & 60 & 358 \\
\hline & Sí & 4 & 1 & 8 \\
\hline \multirow[t]{2}{*}{ Sulfonilureas } & No & 134 & 59 & 359 \\
\hline & Sí & 4 & 2 & 7 \\
\hline
\end{tabular}


sérica normal junto con $\mathrm{FG}>60 \mathrm{~mL} / \mathrm{min} / 1,73 \mathrm{~m}^{2}$, mientras que a la coexistencia de creatinina elevada con $\mathrm{FG}<60$ $\mathrm{mL} / \mathrm{min} / 1,73 \mathrm{~m}^{2}$ se le denominó "insuficiencia renal crónica" (IRC). También se clasificó a los pacientes según los estadios de ERC establecidos por la National Kidney Foundation (NKF) de Estados Unidos (1).

\section{ANÁLISIS ESTADÍSTICO}

Se ha realizado un análisis descriptivo de los datos. Las variables cuantitativas se describen como media \pm 1 desviación estándar (SD) además del intervalo de confianza (CI ) del $95 \%$.

Las variables categóricas fueron analizadas mediante el test de $\chi^{2}$. Se utilizó el test de la "t de Student" en la comparación de variables cuantitativas. Para la comparación de medias de más de dos grupos se utilizó la técnica del análisis de la varianza con contrastes a posteriori (prueba de Scheffé). Se examinó mediante análisis de regresión lineal la existencia de asociación lineal entre las variables cuantitativas mediante el coeficiente de correlación de Sperman y se representaron mediante gráficos de dispersión con la recta de regresión. Se realizó un análisis mediante regresión logística para determinar que variables se asociaban con la presencia de insuficiencia renal oculta, controlando por edad, sexo, creatinina sérica e índice de comorbilidad.

Para la realización de todos estos análisis se utilizó el paquete estadístico SPSS 10.0 (Chicago, Illinois). Un valor $\mathrm{p}<0,05$ fue considerado estadísticamente significativo.

\section{RESULTADOS}

Fueron incluidos 567 pacientes de los cuales 297 (52\%) eran hombres y $270(48 \%)$ eran mujeres. La edad media global fue de 71,12 $\pm 15,50$ años (IC95\% 70,84 - 73,40) con un rango entre 14 y 97 años. La edad media de los hombres fue $71,27 \pm 15,43$ años (IC95\% 69,51 - 73,03) y la de las mujeres $73,06 \pm 15,55$ años (IC95\% 71,20 - 74,92) (p = 0,171). En la tabla I se muestran las características de los pacientes agrupados según la función renal. El grupo con insuficiencia renal oculta (62 pacientes; $10,9 \%$ del total) presentó de modo significativo respecto al grupo con función renal normal (367 pacientes) un índice de comorbilidad y un número de fármacos prescritos más elevados, sin existir diferencias con el grupo con IRC (138 pacientes). Los pacientes con IRO asimismo presentaban una edad superior al grupo con función renal normal. Respecto a la distribución del sexo es destacable que en el grupo con IRO las mujeres representaban un $79 \%$ frente al $21 \%$ de los hombres ( $p<0,0001)$. En los otros dos grupos estos porcentajes estaban más equilibrados. En ningún caso se detectó la coexistencia de creatinina sérica elevada junto con $\mathrm{FG}>60 \mathrm{ml} / \mathrm{mto} / 1,73 \mathrm{~m}^{2}$.

En la figura 1 se muestra el porcentaje de pacientes con IRO según sexo estratificados por grupos de edad. Puede observarse como mayoritariamente son las mujeres las afectadas, sobretodo a partir de los 70 años. En el grupo de edad comprendido entre los 71 y 80 años un $28 \%$ de las mujeres presentaban IRO frente a un $3 \%$ de los hombres. En el grupo de edad a partir de 80 años los porcentajes respectivos fueron 18 para las mujeres y $8 \%$ para los hombres. En la figura 2 se

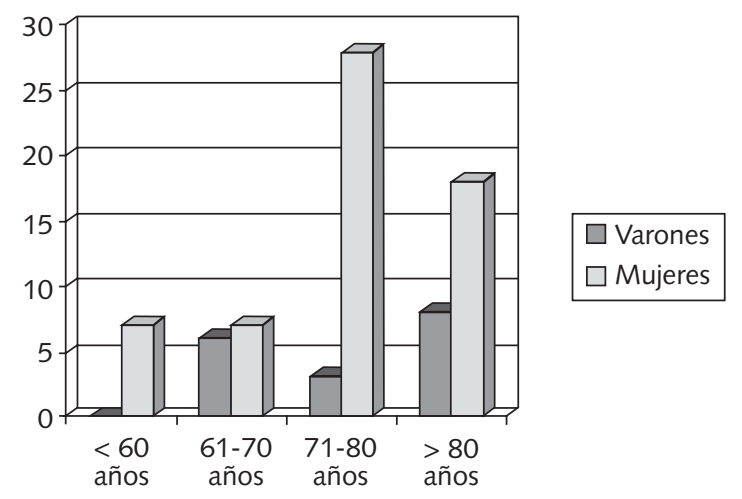

Fig. 1. Porcentaje de IRO según sexo y grupos de edad.

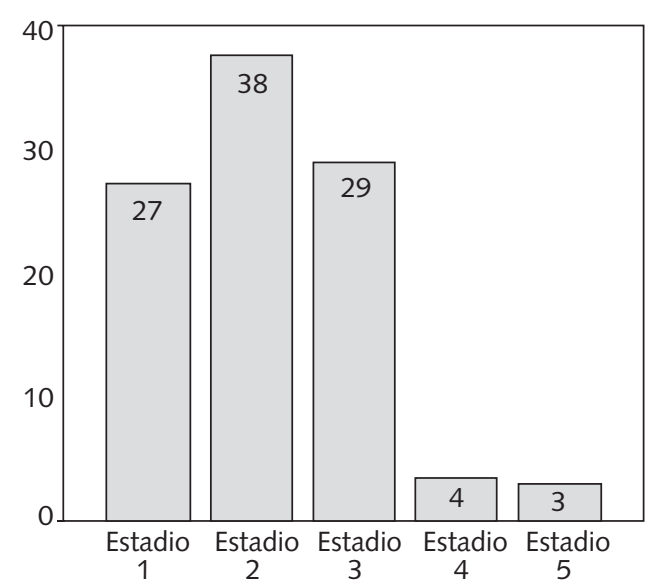

Fig. 2. Estadio de ERC en nuestros pacientes según los criterios de la NKF (estadio 1: aclaramiento de creatinina $>90 \mathrm{ml} / \mathrm{min} / 1,73 \mathrm{~m}^{2}$; estadio 2: aclaramiento de creatinina $60-89 \mathrm{ml} / \mathrm{min} / 1,73 \mathrm{~m}^{2}$; estadio 3: aclaramiento de creatinina 30-59 $\mathrm{ml} / \mathrm{min} / 1,73 \mathrm{~m}^{2}$; estadio 4: aclaramiento de creatinina $15-29 \mathrm{ml} / \mathrm{min} / 1,73 \mathrm{~m}^{2}$; estadio 5: aclaramiento de creatinina $<15 \mathrm{ml} / \mathrm{min} / 1,73 \mathrm{~m}^{2}$ ).

presentan los porcentajes de pacientes en cada estadio de enfermedad crónica clasificados según los criterios de la NKF. El grupo más numeroso lo constituye el estadio 2 (38\%), pero el estadio 3 es el segundo en importancia, con un $29 \%$ de los pacientes. En sólo el 1,4\% de los pacientes ( 8 casos) figuraba en el informe de alta el FG calculado. En todos ellos se llevó a cabo mediante el cálculo del aclaramiento de creatinina tras la recogida de orina de 24 horas y en ningún caso mediante la aplicación de alguna fórmula. En los diagnósticos finales de los informes de alta la insuficiencia renal como tal figuró en el 10,4\% de los pacientes (59 casos).

En el análisis de regresión logística utilizando la presencia de IRO como variable dependiente binaria y controlando por edad, sexo, índice de comorbilidad y creatinina sérica, las variables que permanecieron significativas fueron la edad $(\operatorname{expB}=1,035 ; \mathrm{IC} 95 \% 1,012-1,060 ; \mathrm{p}=0,004)$ y el sexo femenino $(\operatorname{expB}=4,669 ;$ IC95\% 2,461 - 8,856; p < 0,0001).

En la tabla II se muestra el listado de fármacos seleccionados para este estudio que deben ser ajustados según la función renal o administrados con precaución en caso de insuficiencia 
renal, pautados a los pacientes en el informe de alta, estratificados según la presencia de IRO, IRC o función renal normal. Algunos de estos fármacos fueron ampliamente indicados a pacientes con IRO. Destacan los IECA/ARA II (22 pacientes, en 12 de ellos se trataba de IECA), la digoxina (16 pacientes), la espironolactona (7), los AINE (5) y la metformina (4). Por el contrario sólo dos pacientes con IRO recibieron amilorida y tres alopurinol. En los pacientes con IRC estos fármacos también fueron utilizados con profusión (IECA/ARAII 56 casos, 23 de ellos IECA; Digoxina 19; espirolactona 7; alopurinol 19; AINEs 7). Dentro del grupo con IRO 22 pacientes $(35,5 \%)$ no recibieron ningún fármaco del listado, 21 pacientes $(33,8 \%)$ recibieron un solo fármaco y 17 pacientes $(27,4 \%)$ recibieron dos. Finalmente en un solo caso el paciente recibió cuatro de los fármacos y en otro caso fueron cinco.

Mediante análisis de regresión lineal se comprobó la existencia de una asociación lineal positiva entre las cifras de creatinina sérica y el índice de comorbilidad ( $\mathrm{r}=0,240 ; \mathrm{p}<$ $0,0001)$ así como entre la creatinina y el número de fármacos prescritos $(r=0,219 ; \mathrm{p}<0,0001)$ (Fig. 3). También existió asociación lineal entre el índice de comorbilidad y el número de fármacos prescritos $(\mathrm{r}=0,418 ; \mathrm{p}<0,0001)$

\section{DISCUSIÓN}

En nuestro estudio uno de cada diez pacientes ingresado en un servicio de medicina interna presentaba la denominada insuficiencia renal "oculta", es decir, coexistiendo con unas cifras de creatinina sérica dentro del límite normal del laboratorio, el FG estimado de estos pacientes estaba en el rango de la insuficiencia renal $\left(<60 \mathrm{ml} / \mathrm{min} / 1,73 \mathrm{~m}^{2}\right)$. En trabajos publicados previamente este porcentaje de afectados varió en función de las características de la población y de la fórmula aplicada para la estimación del FG (6-12). Como puede apreciarse en la tabla III, la prevalencia se sitúa en los diferentes estudios realizados recientemente en nuestro país en un amplio margen entre el 10 y el $45 \%$ cuando se consideran de modo global las muestras. En trabajos como el de FernándezFresnedo esta prevalencia es incluso mayor cuando se analiza a la población según estratos de edad y sexo. En ese trabajo y de modo similar al nuestro se confirma que este problema afecta sobretodo a personas de edad avanzada con predominio mayoritario del sexo femenino, en nuestra población reafirmado por los resultados del modelo de regresión logística que muestra como las mujeres presentan un riesgo de IRO 4,6 veces superior al de los hombres. Esto es debido a que cuando la población envejece, especialmente en mujeres, se produce una disminución de la masa muscular, con menor producción de creatinina a partir de creatina por el metabolismo del músculo esquelético, lo que condiciona una distorsión muy importante en la relación existente entre la creatinina sérica y el filtrado glomerular. Este fenómeno puede conducir a sobrevalorar la función renal si sólo se estima mediante la creatinina (16).

El desconocimiento de esta patología puede acarrear importantes consecuencias para los pacientes. Si el médico no es consciente de la existencia de una insuficiencia renal el paciente no será remitido al nefrólogo, y cuando la enfermedad renal sea evidente la referencia se producirá de modo tardío. Esta referencia tardía tiene importantes consecuencias en términos de morbimortalidad para los pacientes como

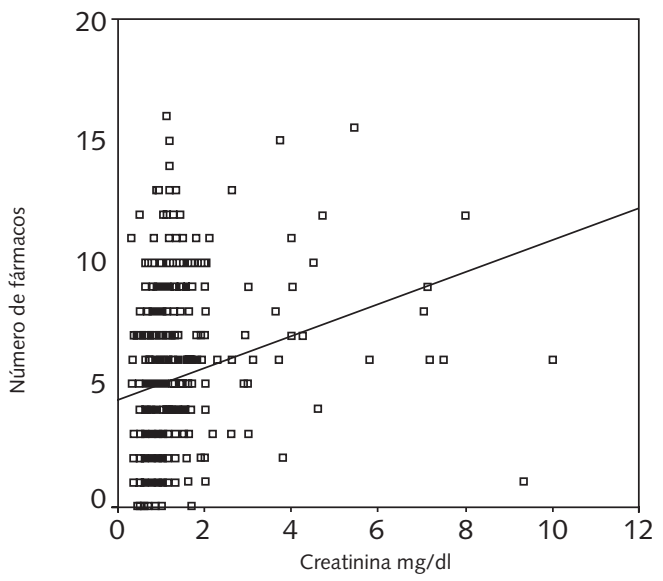

Fig. 3. Asociación entre creatinina sérica y número de fármacos prescritos.

\section{TABLA III}

ESTUDIOS PREVIOS EN LOS QUE SE EVALUÓ LA PREVALENCIA DE IRO

\begin{tabular}{|c|c|}
\hline Autor/año & Prevalencia IRO \\
\hline $\begin{array}{l}\text { Fernández-Fresnedo } \\
\text { G (6) } 2002\end{array}$ & $\begin{array}{l}1.053 \text { pacientes ambulatorios } \quad \text { *Expresada en } \\
\text { con creatinina }<2,5 \mathrm{mg} / \mathrm{dl} \quad \text { función de sexo y edad }\end{array}$ \\
\hline $\begin{array}{l}\text { Otero A (7) } \\
2005\end{array}$ & $\begin{array}{l}1.059 \text { casos }>18 \text { años seleccio- } \\
\text { nados aleatoriamente en un } \\
\text { estudio poblacional }\end{array}$ \\
\hline $\begin{array}{l}\text { Tranche S (9) } \\
2005\end{array}$ & $\begin{array}{l}499 \text { pacientes con diabetes tipo } 2 \\
\text { atendidos en atención primaria }\end{array}$ \\
\hline $\begin{array}{l}\text { Rodrigo MP (8) } \\
2006\end{array}$ & 1.000 pacientes de atención primaria $\quad$ 10,4 (MDRD) \\
\hline $\begin{array}{l}\text { Fernández Vega F (10) } \\
2006\end{array}$ & $\begin{array}{l}2.130 \text { pacientes con HTA esencial } 13,42 \% \text { (MDRD) } \\
\text { del estudio FRESHA. Realizado en } \\
\text { atención primaria }\end{array}$ \\
\hline $\begin{array}{l}\text { Almirall J (11) } \\
2005\end{array}$ & $\begin{array}{l}253 \text { casos > } 64 \text { años seleccionados } \\
\begin{array}{c}\text { aleatoriamente en un estu- } \\
\text { dio poblacional }\end{array} \quad \begin{array}{c}26-45 \% \text { según la } \\
\text { fórmula utilizada }\end{array}\end{array}$ \\
\hline
\end{tabular}

Peña JM

2006

(presente estudio)

567 pacientes ingresados en un $\quad 10,8 \%$ (MDRD)

MDRD: aplicando la fórmula MRDR abreviada; C-G: aplicando la fórmula de Cockcroft-Gault (aclaramiento $=140$ - edad $\times$ peso/creatinina $\times 72$. En caso de sexo femenino multiplicar por 0,85$)$; *: en hombres: $11,3 \%$ entre $70-80$ años; $33,3 \%$ en > 80 años; en mujeres: $35 \%$ entre $70-80$ años; $57 \%$ en > 80 años; **: no se especifica nada más. Se utilizaron las fórmulas MDRD completa y abreviada, así como C-G.

demuestran diversos trabajos publicados (17-20). Muchas de las complicaciones de la insuficiencia renal se establecen desde fases muy tempranas de la misma y es en estos estadios precoces cuando se deben emprender las acciones encaminadas a retrasar la progresión de la enfermedad y prevenir el desarrollo de las mismas (21). Por otra parte la mortalidad de 
los pacientes afectos de enfermedad renal crónica se produce sobretodo por la presentación de eventos cardiovasculares, antes incluso de que la evolución de la enfermedad renal llegue a los estadios finales $(22,23)$. Por ello la detección precoz en atención primaria y el manejo apropiado de los factores de riesgo cardiovascular son fundamentales en estos pacientes.

Otro problema añadido es el de la prescripción de fármacos a esta población. Esta insuficiencia renal "oculta" es fácilmente agravada con la enorme cantidad de medicaciones que interfieren fundamentalmente en la hemodinámica glomerular como son los antiinflamatorios no esteroideos, los inhibidores de la ECA y otro tipo de fármacos. En nuestro estudio hemos comprobado como los pacientes con IRO de modo similar a los afectos de IRC recibieron una cantidad significativamente mayor de principios activos que los pacientes con función renal normal, de hecho existía una asociación lineal positiva entre la creatinina sérica y el número de fármacos. Como muestra la tabla II los pacientes del grupo con IRO fueron tratados en un número importante con fármacos cuya dosificación debe ajustarse según los niveles de aclaramiento renal o que pueden ocasionar efectos deletéreos sobre el funcionalismo renal, sobretodo si este ya está afectado. Además en algunos de estos casos se asociaron más de uno de los fármacos en el mismo paciente. Evidentemente en algunas circunstancias los beneficios que proporcionan algunas de estas medicaciones, como los IECA, superan a los riesgos, pero siempre que el médico conozca la verdadera función renal del paciente y esté alerta sobre la presentación de efectos adversos. No hemos analizado en el estudio la incidencia de estos posibles efectos adversos que se pudieron producir y que pudieron ser atribuidos a determinados fármacos prescritos de modo inadecuado a pacientes con IRO, únicamente hemos descrito la prevalencia del problema.

A pesar de que en 138 casos existían criterios de insuficiencia renal establecida, tan sólo en 59 de ellos la enfermedad figuraba reflejada en los diagnósticos finales del informe de alta y en sólo 8 casos se hacía constar el FG estimado durante el ingreso, siempre mediante la recogida de orina de 24 horas y en ningún caso tras la aplicación de alguna fórmula matemática. Estos datos parecen afirmar que, al menos en nuestro hospital, no existe una concienciación suficiente entre el resto de las especialidades médicas sobre la importancia del tema que estamos tratando. Nos corresponde a los nefrólogos realizar una labor divulgativa en este sentido, tarea a la que esperamos pueda contribuir este trabajo.

La distribución de pacientes según los estadios de la enfermedad crónica en nuestra población mostró un 35,2\% de casos incluidos en los estadios 3, 4, y 5. Lo que indica que en nuestra muestra un tercio de los ingresados presentaban algún grado de insuficiencia renal. Este dato se enmarca en el contexto de una población añosa y con un elevado índice de comorbilidad como la de nuestro estudio. De hecho comprobamos la existencia de una asociación lineal positiva entre la creatinina sérica y el índice de comorbilidad. Es complicado establecer si estos datos tan abultados son generalizables a otros hospitales o más bien son el reflejo de ciertas particularidades de nuestra población.

Una posible limitación de este trabajo es que la fórmula MDRD ha resultado inadecuada en trabajos previos para la estimación del FG en pacientes graves hospitalizados (24), tendiendo a sobreestimar el mismo sobretodo en aquellos pacientes con una ratio BUN (nitrógeno ureico sérico)/creatinina sérica $>20$. No hemos controlado este aspecto pero en todo caso de haberse producido un sesgo en la estimación de nuestra prevalencia de IRO habría sido en el sentido de infraestimarla.

Gran parte de la problemática planteada en este trabajo se podría mitigar si se generalizase en los laboratorios clínicos la estimación del FG a partir de una fórmula en todo paciente al que se solicita una determinación de función renal. En este sentido hay que resaltar la reciente iniciativa de la Sociedad Española de Nefrología dentro de su acción estratégica frente a la enfermedad crónica para la elaboración de un documento de consenso con la Sociedad Española de Química Clínica y Patología Molecular (SEQC) para que se introduzca el filtrado glomerular estimado mediante la fórmula MDRD en todos los informes de los laboratorios españoles $(25,26)$. Esperemos que esta iniciativa fructifique lo que sin duda repercutirá de modo beneficioso en la detección y el manejo de los pacientes renales.

\section{Bibliografía}

1. Levey AS, Coresh J, Balk E, Kausz AT, Levin A, Steffes MW, et al. National Kidney Foundation Practice Guyidelines for Chronic Kidney Disease: Evaluation, Classification and Stratification. Ann Intern Med 2003; 139: 137-47

2. Stevens LA, Coresh J, Greene T, Levey AS. Assessing Kidney function- measured and estimated glomerular filtration rate. N Engl J Med 2006; 354: 2473-83.

3. Cockcroft DW, Gault MG. Prediction of creatinine clearance from serum cretinine. Nephron 1976;16:31-41.

4. Levey AS, Bosch JP, Lewis JB, Greene T, Rogers N, Roth D. A more accurate method to estimate glomerular filtration rate from serum cretinine: a new prediction equation. Modification of Diet in Renal Disease Study Group. Ann Intern Med 1999; 130: 461-70.

5. Levey AS, Greene T, Kusec JW, Beck GJ, Group MS. A simplified equation to predict glomerular filtration rate from serum cretinine. J Am Soc Nephrol 2000; 11: A0828.

6. Fernández Fresnedo G, de Francisco ALM, Rodrigo E, Piñera C, Herráez I, Ruiz C, et al. Insuficiencia renal "oculta” por valoración de la función renal mediante creatinina sérica. Nefrología 2002; 22: 144-51.

7. Otero A, Abelleira A, Gayoso P. Enfermedad renal crónica oculta y factores de riesgo vascular asociados. Estudio epidemilógico. Nefrología 2005; $25: 275-87$.

8. Rodrigo MP, Andres MR. Detección de insuficiencia renal oculta en consulta de atención primaria mediante la aplicación de la ecuación MDRD-abreviada: Análisis de 1000 pacientes. Nefrología 2006; 26: 339-43.

9. Tranche S, Riesgo A, Marín R, Díaz Gonzalez G, García Fernández A. Prevalencia de insuficiencia renal "oculta" en población diabética tipo 2. Aten Primaria 2005; 35: 359-64.

10. Fernández Vega, Riesgo A, Marín R, Herrero P, Prieto MA, Gorostidi $\mathrm{M}$, et al. Insuficiencia renal oculta en pacientes con hipertensión esencial. Estudio FRESHA. Nefrología 2006; 26 (Supl. 6): 21.

11. Almirall J, Vaqueiro M, Antón E, Baré ML, González V, Jaimez E, Gimeno C. Prevalencia de la insuficiencia renal en la población general mayor de 64 años y episodios cardiovasculares asociados. Nefrología $2005 ; 25: 655-62$. 
12. Anandarajah S, Tai T, de Lusignan S, Stevens P, O’Donoghue D, Walker $\mathrm{M}$, et al. The validity of searching routinely collected general practice computer data to identify patients with chronic kidbey disease (CKD): A manual review of 500 medical records. Nephrol Dial Transplant 2005; 20: 2089-96.

13. Pérez García R. Manejo de medicamentos en los enfermos renales. En: Hernando Avendaño, Aljama García P, Arias Rodríguez M, Caramelo Díaz C, Egido de los Ríos J, Lamas Peláez S, editores. Nefrología Clínica ( $1^{\mathrm{a}}$ ed) . Madrid: Editorial Panamericana; 1998. p. 593-602.

14. Olyaei AJ, de Mattos AM, Bennet WM. Prescribing drugs in renal disease. En: Brenner \& Rector's. The Kidney ( $6^{\text {th }}$ ed). Philadelphia: WB Saunders Company; 2000. p. 2606-53.

15. Charlson ME, Pompei P, Ales KL, MacKenzie CR: A new method of classifying prognostic comorbidity in longitudinal studies: Development and validation. J Chronic Dis 1987; 40: 373-83.

16. Duncan L, heathcote J, Djurdjev O, Levin A: Screening for renal disease using serum creatinine: Who are we missing? Nephrol Dial Transplant 2001; 16: 1042-46.

17. Peña JM, Logroño JM, Pernaute R, Laviades C, Virto R, Vicente de Vera C. La referencia tardía al nefrólogo influye en la morbi-mortalidad de los pacientes en hemodiálisis. Un estudio provincial. Nefrología 2006; 26: 84-97.

18. Stack AG: Impact of timing of nephrology referral and pre-ESRD care on mortality risk among new ESRD patients in the united states. Am J Kidney Dis 2003; 41: 310-18.
19. Winkelmayer WC, Owen W, Levin R, Avorn J: A propensity analysis of late versus early nephrologist referral and mortality on dialysis. J Am Soc Nephrol 2003;14: 486-92.

20. Cass A, Cunningham J, Arnold PC, Snelling P, Wang Z, Hoy W. Delayed referral to a nephrologist: Outcomes among patients who survive at least one year on dialysis. Med J Aust 2002; 177: 135-38.

21. De Francisco ALM, Fernández Fresnedo G. Llegada tardía a diálisis como consecuencia de insuficiencia renal no identificada. Nefrología 2002; 22: 95-7.

22. Anavekar NS, McMurray JJV, Velázquez EJ, Solomon SD, Kober L, Rouleau JL, et al. Relation Between renal dysfunction and cardiovascular outcomes after myocardial infarction. N Eng J Med 2004; 351: 1285-95.

23. Go AS, Chertow GM, Fan D, McCulloch CE, Hsu C: Chronic kidney disease and the risk of death, cardiovascular events, and hospitalization. N Eng J Med 2004; 351: 1296-305.

24. Poggio ED, Nef PC, Wang X, Greene T, Van Lente F, Dennis VW, et al. Performance of the Cockcroft-Gault and modification of diet in renal disease equations in estimating GFR in ill hospitalized patients. Am J Kidney Dis 2005; 46: 242-52.

25. Alcázar R, De Francisco ALM. Acción estratégica de la SEN frente a la enfermedad renal. Nefrología 2006; 26: 1-4.

26. Gracia S, Montañés R, Bover J, Cases A, Deulofeu R, De Francisco ALM, et al. Recomendaciones sobre la utilización de ecuaciones para la estimación del filtrado glomerular en adultos. Nefrología 2006; 26: 658-65. 